\title{
Distribution and differential expression of microRNAs in the intestinal mucosal layer of necrotic enteritis induced Fayoumi chickens
}

\author{
Deivendran Rengaraj ${ }^{1}$, Anh Duc Truong', Jihye Ban', Hyun S. Lillehoj', and Yeong Ho Hong ${ }^{1, *}$
}

\footnotetext{
* Corresponding Author: Yeong Ho Hong Tel: +82-31-670-3025, Fax: +82-31-671-3025, E-mail: yhong@cau.ac.kr

1 Department of Animal Science and Technology, Chung-Ang University, Anseong 17546, Korea ${ }^{2}$ Animal Biosciences and Biotechnology Laboratory, Agricultural Research Services, United States Department of Agriculture, Beltsville, MD 20705, USA
}

Submitted Sept 12, 2016; Revised Nov 10, 2016; Accepted Jan 11, 2017
Objective: Despite an increasing number of investigations into the pathophysiology of necrotic enteritis (NE) disease, etiology of NE-associated diseases, and gene expression profiling of NEaffected tissues, the microRNA (miRNA) profiles of NE-affected poultry have been poorly studied. The aim of this study was to induce NE disease in the genetically disparate Fayoumi chicken lines, and to perform non-coding RNA sequencing in the intestinal mucosal layer. Methods: NE disease was induced in the Fayoumi chicken lines (M5.1 and M15.2), and noncoding RNA sequencing was performed in the intestinal mucosal layer of both NE-affected and uninfected chickens to examine the differential expression of miRNAs. Next, quantitative realtime polymerase chain reaction (real-time $\mathrm{qPCR}$ ) was performed to further examine four miRNAs that showed the highest fold differences. Finally, bioinformatics analyses were performed to examine the four miRNAs target genes involvement in the signaling pathways, and to examine their interaction.

Results: According to non-coding RNA sequencing, total 50 upregulated miRNAs and 26 downregulated miRNAs were detected in the NE-induced M5.1 chickens. While 32 upregulated miRNAs and 11 downregulated miRNAs were detected in the NE-induced M15.2 chickens. Results of real-time qPCR analysis on the four miRNAs (gga-miR-9-5p, gga-miR-20b-5p, ggamiR-196-5p, and gga-let-7d) were mostly correlated with the results of RNAseq. Overall, ggamiR-20b-5p was significantly downregulated in the NE-induced M5.1 chickens and this was associated with the upregulation of its top-ranking target gene, mitogen-activated protein kinase, kinase 2. Further bioinformatics analyses revealed that 45 of the gene targets of gga-miR-20b$5 \mathrm{p}$ were involved in signal transduction and immune system-related pathways, and 35 of these targets were predicted to interact with each other.

Conclusion: Our study is a novel report of miRNA expression in Fayoumi chickens, and could be very useful in understanding the role of differentially expressed miRNAs in a NE disease model.

Keywords: Fayoumi Chicken; Necrotic Enteritis; Intestinal Mucosal Layer; microRNA

\section{INTRODUCTION}

Necrotic enteritis (NE) disease that affects broiler chickens is a serious problem for poultry industries. The causative agent of NE disease is a gram-positive bacterium Clostridium perfringens (C. perfringens). NE disease causes intestinal mucosal damage leading to poor digestion and absorption in infected birds. The intestinal mucosal damage permits the pathogen to enter the bile duct and portal veins, and colonize the liver [1]. In addition to C. perfringens, a parasitic protozoa Eimeria maxima (E. maxima) has been isolated from the intestines of NE-affected birds [2]. Therefore, the experimental induction of NE disease in chickens by $C$. perfringens alone, or by coinfection with $C$. perfringens and E. maxima, has become prevalent in veterinary studies [3]. The use of poultry feed containing effective antibiotics is the principal approach used for the control of NE 
disease [4]. Since the first report of NE disease, there has been increasing interest in studying the pathophysiological conditions of NE disease, the etiology of NE-associated diseases, the development of control measures, and the gene expression analyses of the tissues from NE-afflicted chickens [2,4,5]. However, there is currently a deficiency of studies investigating the expression of small non-coding RNAs, especially microRNAs (miRNAs), in NE-affected tissues.

MicroRNAs are small non-coding RNAs that play important roles in the post-transcriptional regulation of genes. The expression and/or regulation of miRNAs are crucial for various biological activities including cell proliferation and differentiation, embryo development, disease progression, and cellular metabolisms. Several studies have reported that miRNAs preferentially bind to the 3'-untranslated region (3'UTR) during post-transcriptional gene regulation [6,7]. miRNAs regulate genes in both normal and disease conditions; however, the expression of miRNAs itself altered in most cancer and other diseases. These changes to miRNA expression in diseases can be caused by alterations involving miRNA loci or the abnormal expression of transcription factors that target miRNAs [8]. To our knowledge, the expression of miRNAs is poorly studied in NE disease models; with the exception of our previous studies on inbred White Leghorn chickens $[9,10]$. The Egypt originated Fayoumi breed was imported to the United States in 1954 . This breed was originally reported to be resistance to the avian leucosis, and later shown to develop a vigorous protective response against Eimeria tenella (E. tenella), which is one of the causative agents of coccidiosis [11,12]. Fayoumi M5.1 and M15.2 chickens are congenic pair of original Fayoumi breed, and highly inbred with an inbreeding coefficient of 0.99 . They share an identical genetic background and differ only in the microchromosome bearing the major histocompatibility complex. These two chicken lines are genetically distant from the broiler and White Leghorn chickens [12,13]. Our earlier studies have demonstrated that the Fayoumi chicken line M5.1 is more resistant to E. maxima/E. tenella when compared to the M15.2 line [14].

The aim of the present study was to examine the distribution and differential expression of miRNAs in the intestinal mucosal layer (IML) of the genetically disparate Fayoumi M5.1 and M15.2 chickens following the induction of NE disease by C. perfringens and $E$. maxima coinfection. By high-throughput small non-coding RNA sequencing, a greater number of miRNAs were differentially expressed in the IML of NE-induced M5.1 chickens, compared to NE-induced M15.2 chickens. Next, four miRNAs (gga-miR9-5p, gga-miR-20b-5p, gga-miR-196-5p, and gga-let-7d) that showed the highest fold differences in the NE-induced chickens were examined by quantitative real-time polymerase chain reaction (real-time qPCR) and annotation of their target genes. Our study is a novel report of miRNA profiles and regulation in Fayoumi chickens with experimentally induced NE.

\section{MATERIALS AND METHODS}

\section{Experimental birds and their care}

Two highly inbred Fayoumi chicken lines (M5.1 and M15.2) obtained from Iowa State University (Ames, IA, USA) were used in the present study. The care and experimental use of these birds was approved by the Beltsville Area Institutional Animal Care and Use Committee of the US Department of Agriculture-Agricultural Research Service (USDA-ARS). The birds' management, the experimental induction of NE, and sample collection were performed at USDA-ARS Beltsville, USA. Subsequent experiments, including high-throughput small RNA sequencing and quantitative realtime polymerase chain reaction analysis, were performed in the Republic of Korea, according to the USDA-ARS and Chung-Ang University agreement (59-1265-0-042F).

\section{Experimental induction of $\mathrm{NE}$ and sample collection}

$\mathrm{NE}$ was experimentally induced in chickens from lines M5.1 and M15.2 by infecting them with E. maxima strain $41 \mathrm{~A}\left(1.0 \times 10^{4}\right.$ oocysts/bird) by oral gavage on post-hatch day 14 , followed by oral gavage with $C$. perfringens strain Del- $1\left(1.0 \times 10^{9}\right.$ colonyforming units/bird) on post-hatch day 18 . The induction of NE was further promoted by feeding the chickens with an antibioticfree organic starter diet containing 17\% crude protein between days 1 and 18, followed by a standard grower diet containing 24\% crude protein between days 18 and 20. The uninfected control chickens were maintained in separate cages and provided with standard feed and water ad libitum. To examine the establishment of NE, fecal samples were collected between post-hatch days 14 and 20 ( 0 to 7 days after E. maxima infection) and the oocyst numbers determined using a McMaster chamber (HK Inc., Tokyo, Japan). Gut lesion scores were determined at posthatch day 20 ( 2 days after $C$. perfringens infection), on a scale of 0 to 4 , by three independent observers in a blinded fashion. The average gut lesion scores of the NE-induced M5.1 and M15.2 chickens selected for RNA sequencing were about 1.9 and 2.3, respectively as described early [12].

The IML was collected from NE-induced and control chickens from M5.1 and M15.2 lines (five each) on post-hatch day 20. The mid-jejunum area of the small intestines in both NE-induced and control chickens was cut longitudinally and washed three times with ice-cold Hanks' balanced salt solution supplemented with $100 \mathrm{U} / \mathrm{mL}$ penicillin and $100 \mathrm{mg} / \mathrm{mL}$ streptomycin (SigmaAldrich, St. Louis, MO, USA). The IML was carefully removed using a cell scraper (Nunc, Thermo Scientific Inc., Waltham, MA, USA). The total RNA was extracted from the samples using TRIzol (Invitrogen, Carlsbad, CA, USA), and purified using an RNeasy Mini Kit (Qiagen, Germantown, MD, USA). Trace DNA in the samples was removed using DNase (Promega, Madison, WI, USA) prior to high-throughput small non-coding RNA sequencing.

High-throughput small non-coding RNA sequencing 
Approximately $5 \mu \mathrm{g}$ of total RNA extracted from the IML of M5.1 and M15.2 NE-induced and control chickens was used by the Theragen Bio Institute (Suwon, Korea) to generate high-throughput small non-coding RNA data, using the HiSeq2000 high-throughput sequencer (Illumina, Inc., San Diego, CA, USA) according to the standard manufacturer protocols. Raw data were processed to obtain clean reads by removing low-quality reads, 5 'primer and 3 'primer contaminants, sequences without the insert tag, poly A tags, and reads that were shorter than 18 nucleotides. To further identify sequence reads with reliable chromosomal locations, all clean reads were mapped onto the reference chicken genome (Gallus gallus v.4) obtained from the UCSC Genome database (University of California, Santa Cruz, CA, USA). Among the clean reads, those with desired sequence lengths between 18 and 30 nucleotides were selected as potential small non-coding RNAs [10].

\section{Prediction of miRNAs and clustering of differentially expressed miRNAs}

The annotation of small non-coding RNAs was performed following standard protocols from the Theragen Bio Institute (Korea). Specifically, miRNAs were identified by aligning the sequence reads with the precursor or mature miRNAs of chickens in miRBase (version 18.0) [15]. Additionally, the possibility of novel miRNAs, and their corresponding secondary structures, was predicted by MIREAP from unannotated reads that did not fall in any small non-coding RNA category. The miRNAs that were differentially expressed in the IML of NE-induced chickens, when compared to their respective controls, were determined based on their fold difference ( $\geq 2$-fold change) and p-value ( $\leq$ 0.01 ). The miRNAs that were significantly upregulated or downregulated in the NE-induced chickens, when compared to their respective controls, were subjected to hierarchical clustering using the Multiexperiment Viewer (MeV) tool obtained from the TM4 microarray software suite [16]. Additionally, the miRNAs that were differentially expressed between the NE-induced chickens were determined and clustered.

Real-time qPCR analysis of differentially expressed miRNAs and their target genes

The expression levels of four miRNAs that differentially expressed in the IML of NE-induced chickens when compared to their respective controls were examined by real-time qPCR. The selected miRNAs were: gga-miR-9-5p, upregulated in NE-induced M5.1 chickens; gga-miR-20b-5p, downregulated in NE-induced M5.1 chickens; gga-miR-196-5p, upregulated in NE-induced M15.2 chickens; and gga-let-7d, downregulated in NE-induced M15.2 chickens. The expression levels of the top-ranking/top-scoring target genes for each of these miRNAs (mitogen-activated protein kinase kinase kinase 3 [MAP3K3], mitogen-activated protein kinase kinase kinase 2 [MAP3K2], aquaporin 4 [AQP4], and insulin-like growth factor 2 mRNA binding protein 3 [IGF2BP3], respectively) were also examined by real-time qPCR. The predicted target genes for each miRNA were identified using the miRDB, an online database for miRNA target prediction and functional annotations [17].

Approximately $2 \mu \mathrm{g}$ of total RNA from the IML of M5.1 and M15.2 control and NE-induced chickens was prepared using TRIzol and purified using an RNeasy Mini Kit. Traces of DNA in the samples were removed using DNase prior to cDNA synthesis for miRNA or target gene amplification. The cDNA for miRNA amplification was synthesized from the total RNA (2 $\mu \mathrm{g})$ using the NCode miRNA First-Strand cDNA Synthesis and qRT-PCR Kit (Invitrogen, USA) according to the manufacturer's protocols. Forward primers for gga-miR-9-5p (5'-TCT TTG GTT ATC TAG CTG TAT GA), gga-miR-20b-5p (5'-CAA AGT GCT CAT AGT GCA GGT AG), gga-miR-196-5p (5'-TAG GTA GTT TCA TGT TGT TGG), and gga-let-7d (5'-AGA GGT AGT GGG TTG CAT AGT) were designed following the guidelines of Agilent Technologies (Santa Clara, CA, USA) and synthesized by Genotech Co. Ltd. (Daejeon, Korea). Real-time qPCR was performed in triplicate with $20 \mu \mathrm{L}$ of reaction mix containing $1 \mu \mathrm{L}$ of cDNA (100 ng), $0.5 \mu \mathrm{L}$ of miRNA-specific forward primer ( $5 \mathrm{pmol}$ ), $0.5 \mu \mathrm{L}$ of NCode universal reverse primer ( $5 \mathrm{pmol}), 10 \mu \mathrm{L}$ of $2 \mathrm{x}$ AMPIGENE qPCR Green Mix Lo-ROX (Enzo Life Sciences Inc., Farmingdale, NY, USA), and $8 \mu \mathrm{L}$ of nuclease free water. The thermal conditions for qPCR were as follows: pre-incubation at $50^{\circ} \mathrm{C}$ for $2 \mathrm{~min}$ and $95^{\circ} \mathrm{C}$ for $2 \mathrm{~min} ; 45$ cycles of 2 -step amplification at $95^{\circ} \mathrm{C}$ for $15 \mathrm{~s}$, and $60^{\circ} \mathrm{C}$ for $30 \mathrm{~s}$; and a final cooling step at $37^{\circ} \mathrm{C}$ for $30 \mathrm{~s}$. The samples were quantified using a Light Cycler 96 Real-time PCR System (Roche Diagnostics, Indianapolis, IN, USA). The expression of miRNAs in the NE-induced samples was calculated after normalizing with chicken U24 small nucleolar RNA (Z48762, Forward primer: 5'-GGG ATG TAA AAA AAT ACT TGC TAT C). The correlation coefficient of miRNAs expression by RNA sequencing and real-time qPCR was further analyzed.

The cDNA for target gene amplification was synthesized from the total RNA $(2 \mu \mathrm{g})$ using a RevertAid First Strand cDNA Synthesis Kit (Thermo Scientific Inc., USA) according to the manufacturer's protocols. Forward $(\mathrm{F})$ and reverse $(\mathrm{R})$ primers for MAP3K3 (XM_015299375, F: 5'-TGA CCC GGA AAT ACA CTC GC; R: 5'-ACA TTC CCA GCA GAG TCA CG), MAP3K2 (XM_015290042, F: 5'-TGA GGG ACC CAC CAG AAA GA; R: 5'-GCG AGT CAC GTT CTC TGT CA), AQP4 (NM_001004765, F: 5'-GTG CCA GTA TGA ACC CTG CT; R: 5'-AAG AGC ACC AGC AAG GAC TG), and IGF2BP3 (NM_001006359, F: 5'-GCC TTG GCA GTT GGA GCT AT; R: 5'-AGC TTG GCA TCT GGT CCT TC) were designed using the Primer3 program (National Center for Biotechnology Information [NCBI] database) and synthesized by Genotech Co. Ltd. Real-time qPCR was performed in triplicate with $20 \mu \mathrm{L}$ of reaction mix containing $1 \mu \mathrm{L}$ of cDNA (100 ng), $0.5 \mu \mathrm{L}$ of gene-specific forward primer ( $5 \mathrm{pmol}), 0.5$ $\mu \mathrm{L}$ of gene-specific reverse primer ( $5 \mathrm{pmol}), 10 \mu \mathrm{L}$ of $2 \mathrm{x}$ AM- 
PIGENE qPCR Green Mix Lo-ROX, and $8 \mu \mathrm{L}$ of nuclease free water. The thermal conditions for $\mathrm{qPCR}$ were as follows: preincubation at $95^{\circ} \mathrm{C}$ for $3 \mathrm{~min} ; 45$ cycles of $95^{\circ} \mathrm{C}$ for $30 \mathrm{~s}, 60^{\circ} \mathrm{C}$ for $30 \mathrm{~s}$, and $72^{\circ} \mathrm{C}$ for $30 \mathrm{~s}$; and a final dissociation cycle at $95^{\circ} \mathrm{C}$ for $10 \mathrm{~s}, 65^{\circ} \mathrm{C}$ for $60 \mathrm{~s}, 97^{\circ} \mathrm{C}$ for $1 \mathrm{~s}$, and $37^{\circ} \mathrm{C}$ for $30 \mathrm{~s}$. The samples were quantified using a Light Cycler 96 Real-time PCR System (Roche Diagnostics, USA). The expression of genes in the NEinduced samples was calculated after normalizing with chicken glyceraldehyde 3-phosphate dehydrogenase (NM_204305, F: TGC TGC CCA GAA CAT CAT CC; R: ACG GCA GGT CAG GTC AAC AA).

Analysis of the pathway involvement and interactions between the target genes of differentially expressed miRNAs The predicted target genes of the four miRNAs that differentially expressed in the IML of NE-induced chickens (gga-miR-9-5p, gga-miR-20b-5p, gga-miR-196-5p, and gga-let-7d) when compared to their respective controls were identified using the miRDB [17]. All target genes for each miRNA were then entered into the Kyoto encyclopedia of genes and genomes (KEGG) database [18] to search for their involvement in various pathways. Preferentially, those genes involved in signal transduction pathways and immune system-related pathways were selected. Next, the interactions of target genes that were involved in these pathways were analyzed using the Search Tool for the Retrieval of Interacting Genes/Proteins (STRING) database program (Version 10.0) [19]. The results were retrieved based on a medium confidence view (score 0.4 ) and using the default prediction methods.

\section{Statistical analysis}

The expression of miRNAs and their target genes in control chickens and NE-induced chickens was analyzed using the Student's $t$ test in the SAS software (SAS Institute, Cary, NC, USA). The significance level of these results was analyzed using the general linear model in the SAS software. Statistical significance was indicated as follows: ${ }^{\star} \mathrm{p}<0.05,{ }^{* *} \mathrm{p}<0.01$, and ${ }^{\star * *} \mathrm{p}<0.001$.

\section{RESULTS AND DISCUSSION}

\section{Sequencing of small non-coding RNAs in the IML of NE-induced Fayoumi chickens}

Initially, high-throughput sequencing of small non-coding RNAs in the IML of control and NE-induced Fayoumi M5.1 and M15.2 chickens was performed using the HiSeq2000 high-throughput sequencer, and followed standard protocols to screen the raw sequencing data. Raw data were processed to obtain clean reads by removing low-quality reads, adaptor-ligated contaminants, sequences without the insert tag, and poly A tags. Sequencing reads shorter than 18 nucleotides were also excluded. After cleaning, there were $28,193,988$ and 28,235,783 reads in the IML of control and NE-induced M5.1 chickens, respectively. There were $30,369,880$ and 28,229,041 reads in the IML of control and NEinduced M15.2 chickens, respectively. These reads were mapped onto the reference chicken genome obtained from the UCSC genome database. The results revealed that $59.7 \%$ and $60.65 \%$ of the total reads were mapped for the control and NE-induced M5.1 chickens, respectively. Approximately 59.41\% and 46.26\% of the total reads were mapped for the control and NE-induced M15.2 chickens, respectively. Similarly, a significant proportion of unique reads in the IML of control and NE-induced Fayoumi chickens was obtained (Table 1). Among the total/unique reads, the most frequent read length was between 22 to 24 nucleotides in our test samples. The total reads returned by high-throughput small RNA sequencing include several categories of non-coding RNAs such as miRNA, transfer RNA (tRNA), ribosomal RNA (rRNA), small nucleolar RNA (snoRNA), small cytoplasmic RNA (scRNA), and small nuclear RNAs (snRNA) [10]. However, we were particularly interested in investigating miRNA distribution and expression in the IML of NE-induced Fayoumi chickens. miRNA is a strong candidate for the regulation of gene expression at the post-transcriptional level [20], and dysregulated miRNA expression has been reported in disease conditions [8]. Furthermore, the annotation of miRNAs is simple compared to the annotation of the other small non-coding RNAs that are involved in the post-transcriptional regulation of gene expression.

Table 1. Raw and processed data of small non-coding RNA sequencing in the intestinal mucosal layer of the control and NE-induced Fayoumi chicken lines

\begin{tabular}{|c|c|c|c|c|}
\hline \multirow{2}{*}{ Items } & \multicolumn{2}{|c|}{ Line M5.1 } & \multicolumn{2}{|c|}{ Line M15.2 } \\
\hline & Control & NE-induced & Control & NE-induced \\
\hline Raw reads & $32,136,171$ & $29,109,954$ & $31,701,148$ & $34,776,702$ \\
\hline Total reads after cleaning & $28,193,988$ & $28,235,783$ & $30,369,880$ & $28,229,041$ \\
\hline Total reads mapping to genome & $16,830,404$ & $17,123,992$ & $18,043,613$ & $13,059,227$ \\
\hline Percent total reads mapping to genome (\%) & 59.7 & 60.65 & 59.41 & 46.26 \\
\hline Unique reads & $1,217,612$ & $1,091,181$ & $1,603,013$ & 926,323 \\
\hline Unique reads mapping to genome & 168,607 & 196,970 & 457,345 & 79,777 \\
\hline Percent unique reads mapping to genome (\%) & 13.85 & 18.05 & 28.53 & 8.61 \\
\hline Frequent length of total/unique reads (bp) & 22 & 24 & 24 & 23 \\
\hline Percent frequent length of total/unique reads (\%) & 16.40 & 22.81 & 15.68 & 16.50 \\
\hline
\end{tabular}

$\mathrm{NE}$, necrotic enteritis. 
Table 2. Distribution of known miRNAs in the intestinal mucosal layer of the control and NE-induced Fayoumi chicken lines

\begin{tabular}{|c|c|c|c|c|c|}
\hline Sample library & miRNA & miRNA-5p & miRNA-3p & Total known miRNAs & $\begin{array}{c}\text { Novel miRNA } \\
\text { candidates }\end{array}$ \\
\hline Control line M5.1 & 34 & 62 & 56 & 152 & 116,809 \\
\hline NE-induced line M5.1 & 35 & 62 & 60 & 157 & 95,053 \\
\hline Control line M15.2 & 24 & 49 & 46 & 119 & 112,573 \\
\hline NE-induced line M15.2 & 26 & 60 & 58 & 144 & 151,737 \\
\hline
\end{tabular}

$\mathrm{NE}$, necrotic enteritis.

To identify the distribution of miRNAs across our test samples, the total reads obtained from the IML of control and NE-induced M5.1 and M15.2 chickens were aligned with precursor or mature chicken miRNAs using miRBase. According to miRBase source, 152 known miRNAs in control M5.1 chickens and 157 known miRNAs in NE-induced M5.1 chickens were found. In the M15.2 line, approximately 119 miRNAs and 144 miRNAs were found in control and NE-induced chickens, respectively (Table 2). These results indicate that there are slightly more miRNAs in the IML of M5.1 chickens, when compared to M15.2 chickens. Specifically, the number of known miRNAs was found to be higher in the NE-induced M5.1 chickens. There are clear instructions for identifying or naming miRNAs. Briefly, when two miRNAs originate from the same precursor, the predominantly expressed sequence is named as miRNA and the non-predominant sequence is named as miRNA* (from the opposite arm of the precursor). When the evidence is insufficient to determine which of the two miRNAs is predominant, they are named as miRNA from the 5'arm (miRNA-5p) and miRNA from the 3'arm (miRNA-3p) [21]. In this study, several sequencing reads from all test samples were found to be matched with known miRNAs, miRNA-5p, and miRNA-3p, but no reads were matched with miRNA*. The unannotated reads from all test samples were further examined using MIREAP to identify potentially novel miRNAs and their secondary structures. In total, 116,809 and 95,053 reads were found to be novel miRNA candidates in the control and NE-induced M5.1 chickens, respectively. Similarly, 112,573 and 151,737 reads were found to be novel miRNA candidates in the control and NEinduced M15.2 chickens, respectively.

Since several known miRNAs distributed across our test samples, we aimed to identify differentially expressed miRNAs in the IML of NE-induced M5.1 and M15.2 chickens, when compared to their respective controls. After setting the fold difference $(\geq 2$ fold change) and p-value ( $\leq 0.01), 50$ upregulated miRNAs and 26 downregulated miRNAs in the NE-induced M5.1 chickens was found, when compared to the control (Supplementary Table S1). On the other hand, 32 upregulated miRNAs and 11 downregulated miRNAs in the NE-induced M15.2 chickens was found, when compared to the control (Supplementary Table S2). These results reveal that the total number of differentially expressed miRNAs/upregulated miRNAs is higher in the NE-induced M5.1 chickens than in the NE-induced M15.2 chickens. Figure 1 shows the hierarchical clustering of differentially expressed miRNAs in the IML of NE-induced M5.1 (Figure 1A) and M15.2 (Figure 1B) chickens. When we compared the treatment groups (NEinduced M5.1 chickens and NE-induced M15.2 chickens), 29 upregulated miRNAs in the NE-induced M15.2 chickens and 63 upregulated miRNAs in the NE-induced M5.1 chickens was found (Figure 1C, Supplementary Table S3). This result reinforce that the M5.1 chickens are more resistant to help upregulation of miRNAs that are crucial for the post-transcriptional regulation of gene expression. Upregulation of miRNAs potentially leads to downregulation of the genes that cause harmful effects to the cellular processes.

\section{Target genes of differentially expressed miRNAs}

In high-throughput miRNA sequencing studies, identifying the target genes of miRNAs is helpful for their further analysis and annotation. The target genes of miRNAs can be identified by various computational methods primarily based on sequence complementarity search. In this study, the predicted target genes of miRNAs that were differentially expressed in the IML of NEinduced chickens (Supplementary Tables S1-S3) were identified using miRDB [17]. Next, four miRNAs that showed the highest fold-changes in the NE-induced chickens, compared to their respective controls, was selected for further analysis and annotation. The selected miRNAs were: gga-miR-9-5p (upregulated 37.6 fold in the IML of NE-induced M5.1 chickens), gga-miR20b-5p (downregulated 35.27 fold in the IML of NE-induced M5.1 chickens), gga-miR-196-5p (upregulated 36.03 fold in the IML of NE-induced M15.2 chickens), and gga-let-7d (downregulated 36.8 fold in the IML of NE-induced M15.2 chickens). The miRNA gga-miR-9-5p targets 260 genes, with the top-ranking/ top-scoring gene being MAP3K3 (target rank 1, score 100). The miRNA gga-miR-20b-5p targets 581 genes, with the top-ranking/ top-scoring gene being MAP3K2 (target rank 1, score 100). The miRNA gga-miR-196-5p targets 92 genes, with the top-ranking/ top-scoring gene being AQP4 (target rank 1, score 99). Finally, the miRNA gga-let-7d targets 207 genes, with the top-ranking/ top-scoring gene being IGF2BP3 (target rank 1, score 100).

\section{Real-time qPCR analysis of differentially expressed miRNAs and their target genes}

Real-time qPCR was used to further examine the expression of the four miRNAs (gga-miR-9-5p, gga-miR-20b-5p, gga-miR196-5p, and gga-let-7d) that showed the highest fold-changes 
A

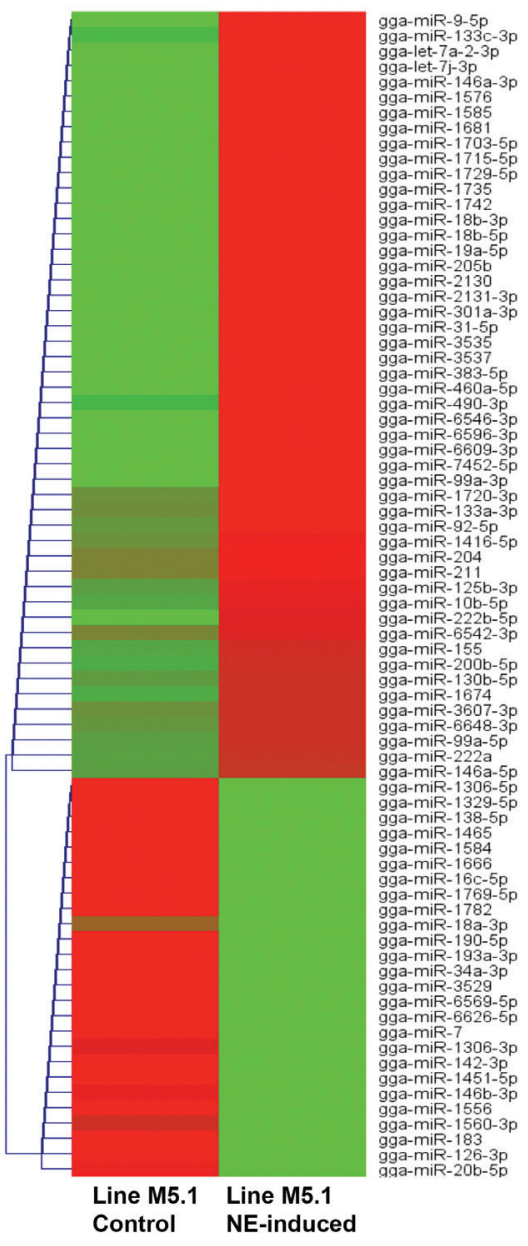

B

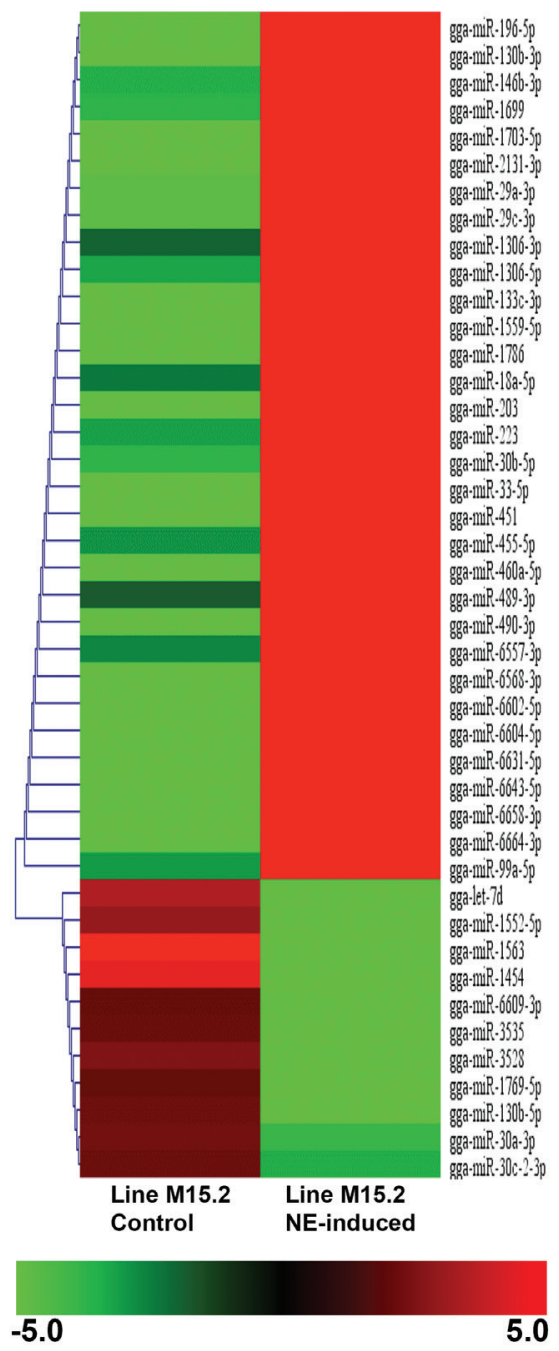

C

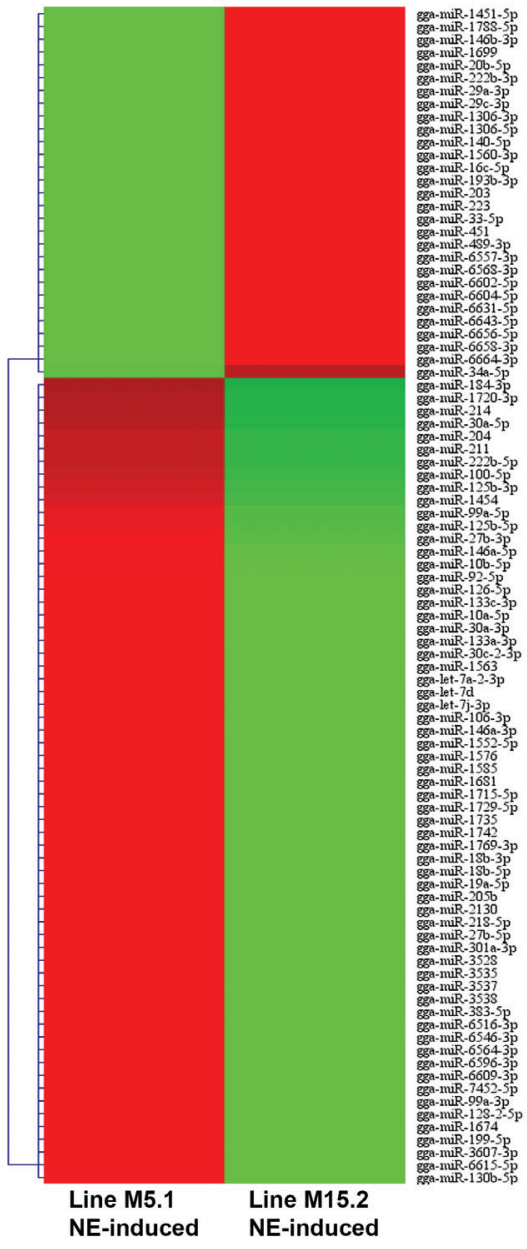

Figure 1. Clustering of miRNAs differentially expressed in the intestinal mucosal layer (IML) of necrotic enteritis (NE)-induced Fayoumi chickens. Hierarchical clustering of miRNAs that were upregulated and downregulated in the IML of NE-induced Fayoumi chicken lines M5.1 (A) and M15.2 (B) after normalization with their respective controls was prepared using the MeV tool. Upregulated and downregulated miRNAs are colored red and green, respectively (Please see online for color). Each row represents the expression of an miRNA, and each column represents a sample.

in the IML of NE-induced chickens, compared to their respective controls, as well as the expression of their top-ranking target genes (MAP3K3, MAP3K2, AQP4, and IGF2BP3, respectively) (Figure 2). The miRNAs gga-miR-9-5p and gga-miR-20b-5p and their target genes $M A P 3 K 3$ and $M A P 3 K 2$, respectively, were amplified with miRNA- or gene-specific cDNAs prepared from the IML of control and NE-induced M5.1 chickens. The miRNAs gga-miR-196-5p and gga-let-7d and their target genes AQP4 and IGF2BP3, respectively, were amplified with miRNA- or genespecific cDNAs prepared from the IML of control and NE-induced M15.2 chickens. The expression of gga-miR-9-5p in the IML of NE-induced M5.1 chickens was not as expected based on the sequencing results. The expression of its target gene MAP $3 K 3$ showed significant increase in the NE-induced chickens compared to the control. The expression of gga-miR-20b-5p was signifi- cantly decreased, and the expression of its target gene MAP3K2 significantly increased, in the IML of NE-induced M5.1 chickens. The expression of gga-miR-196-5p was significantly increased, and the expression of its target gene $A Q P 4$ significantly decreased, in the IML of NE-induced M15.2 chickens. The expression of gga-let-7d was slightly decreased, and the expression of its target gene IGF2BP3 was increased, in the IML of NE-induced M15.2 chickens (Figure 2). The miRNAs expression trends determined by RNA sequencing and real-time qPCR were mostly consistent, with a correlation coefficient of $R^{2}=0.82549$ (Figure 3).

Several studies have reported that the overexpression of miRNAs downregulates their target genes, while downregulation of miRNAs upregulates their target genes, owing to their posttranscriptional gene regulatory functions [7]. This hypothesis is somewhat supported in the case of gga-miR-9-5p, gga-miR- 
A

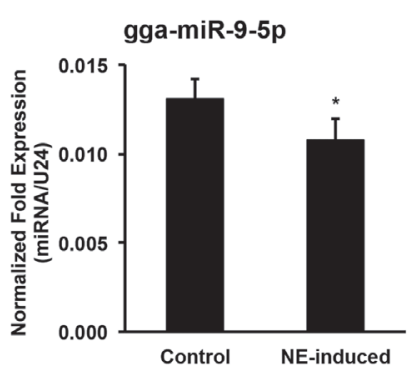

МАРЗКЗ

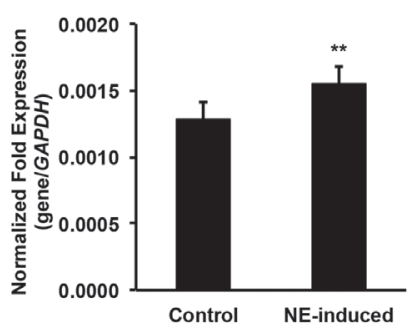

B

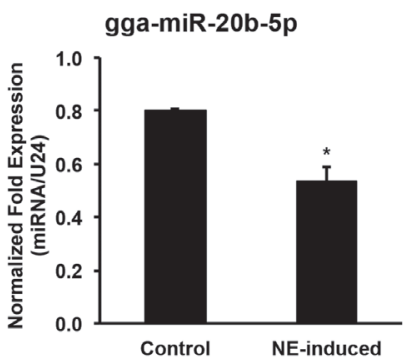

MAP3K2

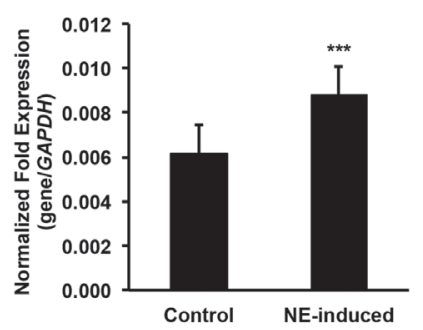

C

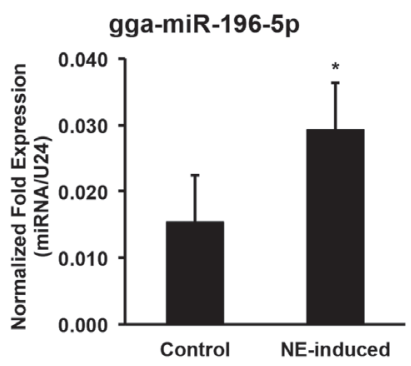

AQP4

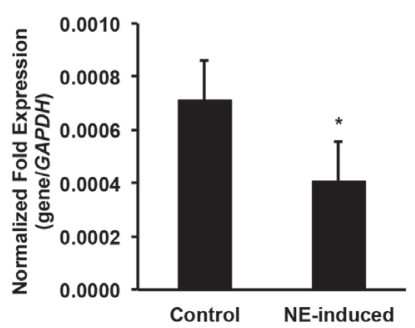

D

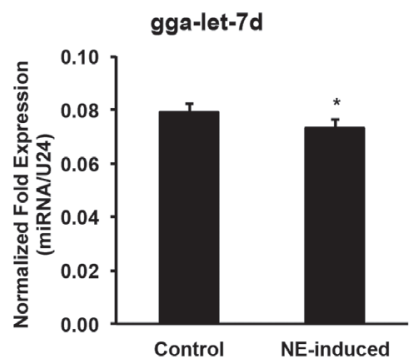

IGF2BP3

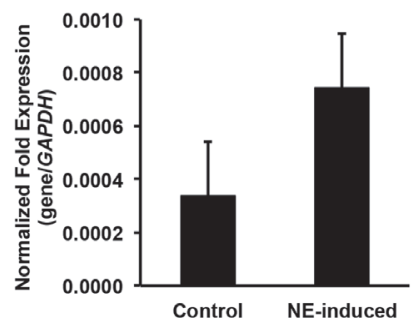

Figure 2. Quantitative real-time polymerase chain reaction analysis of miRNAs differentially expressed in the intestinal mucosal layer (IML) of necrotic enteritis (NE)-induced Fayoumi chickens along with their top ranked target genes. CDNA templates for the amplification of miRNAs and genes were synthesized separately, and amplified with appropriate miRNAand gene-specific primers. (A), (B) miRNAs and genes were amplified with cDNAs from the IML of NE-induced Fayoumi chicken line M5.1. (C), (D) miRNAs and genes were amplified with cDNAs from the IML of NE-induced Fayoumi chicken line M15.2. The expression of miRNAs/genes in NE-induced samples was calculated after normalizing with chicken snoRNA/ glyceraldehyde 3-phosphate dehydrogenase expression. Statistical significance: ${ }^{*} p<0.05,{ }^{* *} p<0.01$, and ${ }^{* * *} p<0.001$.

20b-5p, gga-miR-196-5p, and gga-let-7d and the expression of their target genes MAP3K3, MAP3K2, AQP4, and IGF2BP3, respectively in the IML of NE-induced M5.1 chickens. In miRNA studies, it is important to know whether the expression and functions of selected miRNAs have been reported previously. Among the four miRNAs selected for this study, the expression and functions of miR-9-5p, miR-196-5p, and let-7d had not been previously reported in chickens. A recent study in chicken reported that miR-20b-5p involved in the proliferation and differentiation of chicken myoblast [22]. In humans and/or mice, the expression and functions of miR-9-5p, miR-20b-5p, miR-196-5p, and let-7d have been reported in various disease conditions. Among the four miRNA target genes, the expression and functions of $M A P 3 K 3$, $M A P 3 K 2$, and IGF2BP3 had not been previously reported in

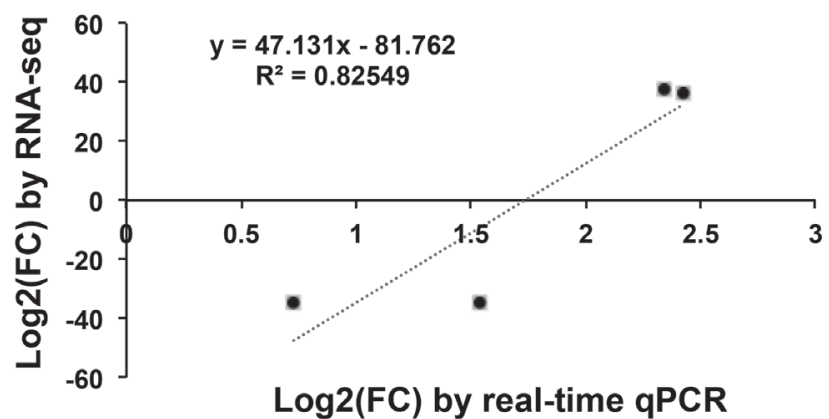

Figure 3. Comparison of the $\log 2$ fold change (FC) of miRNAs differentially expressed in the intestinal mucosal layer (IML) of necrotic enteritis (NE)-induced Fayoumi chickens examined by RNA sequencing and quantitative real-time polymerase chain reaction. chickens. $A Q P 4$ is a member of the water channel family characterized by high water permeability, and its expression was detected in several chicken tissues including gastro-intestinal tract [23]. In humans and/or mice, MAP3K family members MAP3K3 and $M A P 3 K 2$ were reported as the regulators of the NF- $\mathrm{kB}$ and MAPK pathways, and play an important role in enhancing the immune response in the microenvironment of the infected cells [24,25]. Since these two genes were found upregulated in the NE-induced M5.1 chickens, they might involve in enhancing the immune response in this resistant line. The immune related AQP4 autoantibodies positively involved in an inflammatory disease of the central nervous system in humans [26]. Since the AQP4 gene was found downregulated in the NE-induced M15.2 chickens, there might be a clue that this gene decreases the immune response in this susceptible line. IGF2BP3 was reported to decrease the activity of immune cells, and blocking the expression of this gene could increase the fighting ability of immune system [27]. Since IGF2BP3 was found upregulated in the NE-induced M15.2 chickens, it might decrease the immune response in this susceptible line.

\section{Analysis of the pathway involvement and interactions} between the target genes of differentially expressed miRNAs The predicted target genes of the four miRNAs (gga-miR-9-5p, gga-miR-20b-5p, gga-miR-196-5p, and gga-let-7d) that showed the highest fold-changes between the IML of NE-induced chickens and their respective controls, in sequencing analyses, were first retrieved from the miRDB database. According to miRDB, 
gga-miR-9-5p targets 260 genes (target score: 100-50), gga-miR20b-5p targets 581 genes (target score: 100-50), gga-miR-196$5 \mathrm{p}$ targets 92 genes (target score: $99-50$ ), and gga-let-7d targets 207 genes (target score: 100-50). All target genes for each miRNA were then entered into the KEGG database [18] to search for their involvement in pathways identified in chickens. KEGG returned various chicken pathways in relation to these target genes for each miRNA. The intestinal mucosa is one of the most important tissues for the generation of the innate immune response against infectious pathogens that enter through the gastrointestinal tract [28]. Therefore, our analyses was restricted to find the miRNA target genes involved specifically in immune system-related pathways, and signal transduction pathways that essentially support the immune response $[29,30]$. In our results, the target genes of gga-miR-9-5p were found in eight signal transduction pathways and three immune system-related pathways (Figure 4A). In particular, maximum four genes were involved in the phosphatidylinositol signaling system when compared to the number of genes involved in other pathways. The full list of gga-miR-9-5p target genes that are involved in signal transduction and immune system-related pathways are shown in Supplementary Table S4. The target genes of gga-miR-20b-5p were found in 12 signal transduction pathways and four immune system-related pathways, with the maximum of 16 genes in MAPK signaling pathway (Figure 4B, Supplementary Table S5). The target genes of ggamiR-196-5p were found in eight signal transduction pathways and two immune system-related pathways, with the maximum of two genes in the calcium signaling pathway (Figure 4C, Supplementary Table S6). Finally, the target genes of gga-let-7d were found in nine signal transduction pathways, with the maximum of five genes in the MAPK signaling pathway (Figure 4D, Supplementary Table S7).
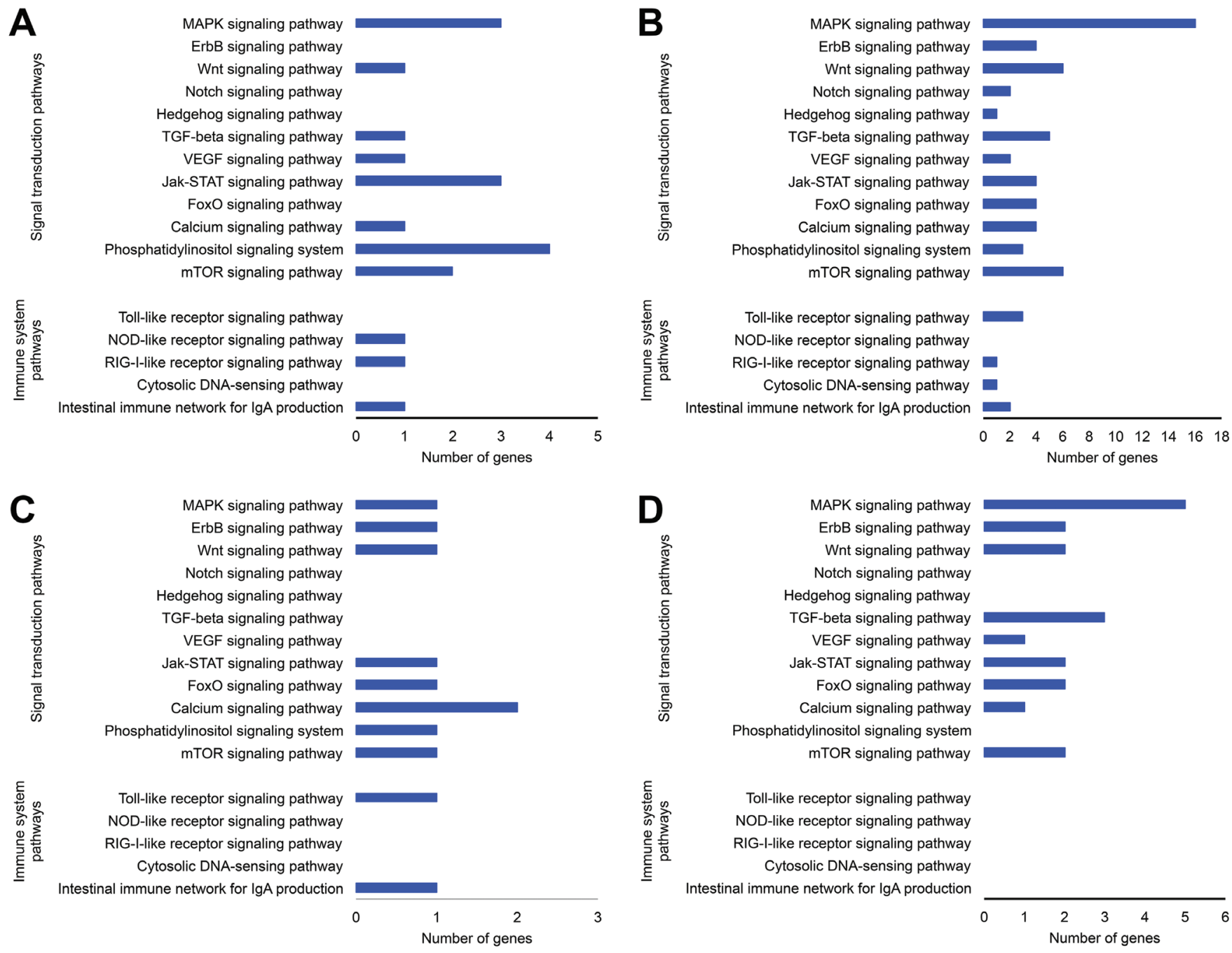

Figure 4. miRNA target genes identified in the signal transduction and immune system-related pathways. The target genes of miRNAs differentially expressed in the intestinal mucosal layer (IML) of necrotic enteritis (NE)-induced Fayoumi chicken lines M5.1 and M15.2 were searched from miRDB, and subjected to the Kyoto encyclopedia of genes and genomes (KEGG) database to identify their involvement in the signal transduction pathways and immune system-related pathways. (A) gga-miR-9-5p target gene pathways. (B) ggamiR-20b-5p target gene pathways. (C) gga-miR-196-5p target gene pathways. (D) gga-let-7d target gene pathways. 
Next, the target genes of gga-miR-9-5p, gga-miR-20b-5p, ggamiR-196-5p, and gga-let-7d that were found to be involved in chicken signal transduction and immune system-related pathways were entered into the STRING database program [19] to find their interactions. The STRING database program enables the analysis of gene/protein-protein interactions in many species including chickens $[19,30]$. The gene interaction results based on a medium confidence view (score 0.4 ) and using the default prediction methods, which included neighborhood, gene fusion, co-occurrence, co-expression, experiments, databases, and textmining were retrieved. In total, 17 gene targets of gga-miR-9-5p were involved in signal transduction and immune system-related pathways; however, only seven genes showed interactions (Figure 5A). Among the eight target genes of gga-miR-196-5p that were involved in signal transduction and immune system-related pathways, none of the genes showed interactions with any of the others (Figure 5C). Among the 15 target genes of gga-let-7d that were involved in signal transduction and immune system-related pathways, only three genes showed interactions (Figure 5D). By contrast, of the 45 gga-miR-20b-5p target genes involved in signal transduction and immune system-related pathways, about 35 genes showed confident interactions (Figure 5B). In the STRING database program, interactions include physical and functional associations that are mainly derived from genomic context, highthroughput experiments, co-expression, and PubMed literature [19]. Overall, the miRNA gga-miR-20b-5p target genes were involved in more signal transduction and immune system-related pathways in the KEGG database and showed more confident interactions in STRING database. The principal reason underlying this finding could be that there were more than 500 target genes for gga-miR-20b-5p, compared with much fewer target genes for the other differentially expressed miRNAs in the IML of NE-induced chickens.

\section{CONCLUSION}

The present study examined the distribution and differential expression of miRNAs in the IML of two genetically disparate Fayoumi chicken lines, M5.1 and M15.2, in which NE disease was experimentally induced. By high-throughput small RNA sequencing, a greater number of miRNAs were differentially expressed in the NE-induced M5.1 chickens (76 miRNAs). The differentially expressed miRNAs in the NE-induced M15.2 chickens were only 43. Then, four miRNAs (gga-miR-9-5p, gga-miR-

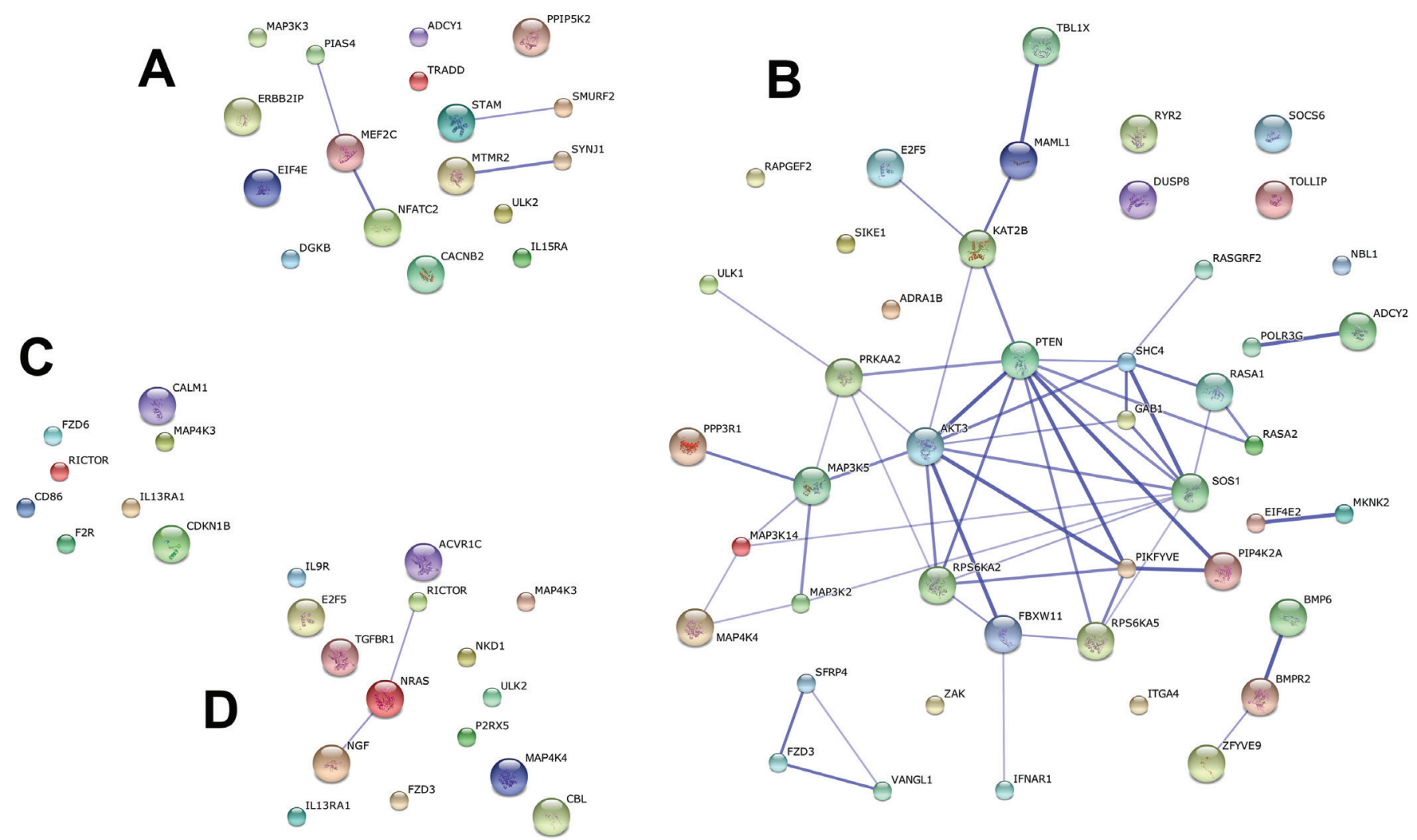

Figure 5. Interaction between miRNA target genes involved in the signal transduction and immune system-related pathways. The miRNAs target genes involved in the signal transduction pathways and immune system-related pathways were subjected to the Search Tool for the Retrieval of Interacting Genes/Proteins (STRING) database program to identify their interactions (medium confidence view with default prediction methods). (A) gga-miR-9-5p target gene interactions. (B) gga-miR-20b-5p target gene interactions. (C) gga-miR196-5p target gene interactions. (D) gga-let-7d target gene interactions. Thicker lines represent stronger interactions, and thinner lines represent medium interactions. Please see abbreviation section for gene descriptions. 
20b-5p, gga-miR-196-5p, and gga-let-7d) that showed the highest fold differences in the NE-induced chickens, compared to the respective controls, were selected for further confirmation by real-time qPCR and bioinformatics-based annotation. When compared to the overall results, the miRNA gga-miR-20b-5p was significantly downregulated in the IML of NE-induced M5.1 chickens and its top-ranking target gene MAP3K2 was significantly upregulated, indicating the miRNAs role in post-transcriptional gene regulation. This miRNA targets 581 genes, and several of these target genes were found to interact with each other and be involved in signal transduction and immune system-related pathways. Since there have been no previous reports of the expression and/or functions of the miRNAs investigated here, our study could be very useful in understanding the role of differentially expressed miRNAs in a NE disease model.

\section{AUTHOR CONTRIBUTIONS}

$\mathrm{DR}$ and YHH conceived and designed the experiments. DR, $\mathrm{ADT}$, and JB performed the experiments and analyzed the data. HSL contributed for the research materials, and data analysis and interpretation. DR wrote the paper. All authors read and approved the final manuscript.

\section{CONFLICT OF INTEREST}

We certify that there is no conflict of interest with any financial organization regarding the material discussed in the manuscript.

\section{ACKNOWLEDGMENTS}

This research was supported by the National Research Foundation grant (NRF-2010-0009360) and the Chung-Ang University research grant in 2016, Republic of Korea.

\section{REFERENCES}

1. Timbermont L, Haesebrouck F, Ducatelle R, Van Immerseel F. Necrotic enteritis in broilers: an updated review on the pathogenesis. Avian Pathol 2011;40:341-7.

2. Broussard CT, Hofacre CL, Page RK, Fletcher OJ. Necrotic enteritis in cage-reared commercial layer pullets. Avian Dis 1986;30:617-9.

3. Jang SI, Lillehoj HS, Lee SH, et al. Vaccination with Clostridium perfringens recombinant proteins in combination with Montanide ISA 71 VG adjuvant increases protection against experimental necrotic enteritis in commercial broiler chickens. Vaccine 2012;30:5401-6.

4. Dahiya JP, Wilkie DC, Van Kessel AG, Drew MD. Potential strategies for controlling necrotic enteritis in broiler chickens in post-antibiotic era. Anim Feed Sci Technol 2006;129:60-88.

5. Oh ST, Lillehoj HS. The role of host genetic factors and host immunity in necrotic enteritis. Avian Pathol 2016;45:313-6.

6. Sun W, Li YSJ, Huang HD, Shyy JYJ, Chien S. microRNA: A master regulator of cellular processes for bioengineering systems. Annu Rev Biomed Eng 2010;12:1-27.

7. Rengaraj D, Park TS, Lee SI, et al. Regulation of glucose phosphate isomerase by the 3'UTR-specific miRNAs miR-302b and miR-17-5p in chicken primordial germ cells. Biol Reprod 2013;89:33.

8. Croce CM. Causes and consequences of microRNA dysregulation in cancer. Nat Rev Genet 2009;10:704-14.

9. Dinh H, Hong YH, Lillehoj HS. Modulation of microRNAs in two genetically disparate chicken lines showing different necrotic enteritis disease susceptibility. Vet Immunol Immunopathol 2014;159:74-82.

10. Hong YH, Dinh H, Lillehoj HS, Song KD, Oh JD. Differential regulation of microRNA transcriptome in chicken lines resistant and susceptible to necrotic enteritis disease. Poult Sci 2014;93:1383-95.

11. Pinard-Van Der Laan MH, Monvoisin JL, Pery P, Hamet N, Thomas M. Comparison of outbred lines of chickens for resistance to experimental infection with coccidiosis (Eimeria tenella). Poult Sci 1998;77: 185-91.

12. Kim DK, Lillehoj HS, Jang SI, et al. Genetically disparate Fayoumi chicken lines show different response to avian necrotic enteritis. J Poult Sci 2015;52:245-52.

13. Zhou H, Lamont SJ. Genetic characterization of biodiversity in highly inbred chicken lines by microsatellite markers. Anim Genet 1999;30: 256-64.

14. Kim DK, Kim CH, Lamont SJ, Keeler CL, Jr., Lillehoj HS. Gene expression profiles of two B-complex disparate, genetically inbred Fayoumi chicken lines that differ in susceptibility to Eimeria maxima. Poult Sci 2009;88:1565-79.

15. Kozomara A, Griffiths-Jones S. miRBase: annotating high confidence microRNAs using deep sequencing data. Nucleic Acids Res 2014;42: D68-73.

16. Saeed AI, Sharov V, White J, et al. TM4: a free, open-source system for microarray data management and analysis. Biotechniques 2003;34: 374-8.

17. Wong N, Wang X. miRDB: an online resource for microRNA target prediction and functional annotations. Nucleic Acids Res 2015;43: D146-52.

18. Kanehisa M, Sato Y, Kawashima M, Furumichi M, Tanabe M. KEGG as a reference resource for gene and protein annotation. Nucleic Acids Res 2016;44:D457-62.

19. Szklarczyk D, Franceschini A, Wyder S, et al. STRING v10: proteinprotein interaction networks, integrated over the tree of life. Nucleic Acids Res 2015;43:D447-52.

20. Bartel DP. MicroRNAs: genomics, biogenesis, mechanism, and function. Cell 2004;116:281-97.

21. Ambros V, Bartel B, Bartel DP, et al. A uniform system for microRNA annotation. RNA 2003;9:277-9.

22. Luo W, Li G, Yi Z, Nie Q, Zhang X. E2F1-miR-20a-5p/20b-5p autoregulatory feedback loop involved in myoblast proliferation and differentiation. Sci Rep 2016;6:27904.

23. Yoshimura K, Sugiura K, Ohmori Y, Aste N, Saito N. Immunolocalization of aquaporin-4 in the brain, kidney, skeletal muscle, and gastro-intestinal tract of chicken. Cell Tissue Res 2011;344:51-61. 
24. Winsauer G, Resch U, Hofer-Warbinek R, Schichl YM, de Martin R. XIAP regulates bi-phasic NF-kappaB induction involving physical interaction and ubiquitination of MEKK2. Cell Signal 2008;20:2107-12.

25. He Y, Wang L, Liu W, et al. MAP3K3 expression in tumor cells and tumor-infiltrating lymphocytes is correlated with favorable patient survival in lung cancer. Sci Rep 2015;5:11471.

26. Li L, Zhang H, Varrin-Doyer M, Zamvil SS, Verkman AS. Proinflammatory role of aquaporin-4 in autoimmune neuroinflammation. FASEB J 2011;25:1556-66.

27. Schmiedel D, Tai J, Yamin R, et al. The RNA binding protein IMP3 facilitates tumor immune escape by downregulating the stress-induced ligands ULPB2 and MICB. Elife 2016;5.
28. Shaughnessy RG, Meade KG, Cahalane S, et al. Innate immune gene expression differentiates the early avian intestinal response between Salmonella and Campylobacter. Vet Immunol Immunopathol 2009; 132:191-8.

29. Truong AD, Hong YH, Lillehoj HS. RNA-seq profiles of immune related genes in the spleen of necrotic enteritis-afflicted chicken lines. Asian-Australas J Anim Sci 2015;28:1496-511.

30. Rengaraj D, Truong AD, Lee SH, Lillehoj HS, Hong YH. Expression analysis of cytosolic DNA-sensing pathway genes in the intestinal mucosal layer of necrotic enteritis-induced chicken. Vet Immunol Immunopathol 2016;170:1-12. 